\title{
Facile electrodeposited NiMoSe nanospheres for hydrogen evolution reaction
}

\author{
Komal Patil $^{\mathrm{a}}$, Pravin Babar ${ }^{\mathrm{b}}$, Xue Li ${ }^{\mathrm{c}}$, Jun Sung Jang ${ }^{\mathrm{a}}$, Dongmyeong Kim ${ }^{\mathrm{a}}$, Myeong Cheol Baek ${ }^{\mathrm{a}}$, \\ Pravin Bhoite ${ }^{\mathrm{d}}$, Jin Hyeok Kim ${ }^{\mathrm{a}}$,* \\ ${ }^{a}$ Optoelectronic Convergence Research Center, Department of Materials Science and Engineering, Chonnam National University, Gwangju 500-757, South Korea \\ ${ }^{\mathrm{b}}$ KAUST Catalysis Center (KCC), Physical Sciences and Engineering (PSE), King Abdullah University of Science and Technology (KAUST), Thuwal 23955, Saudi Arabia \\ ${ }^{\mathrm{c}}$ Department of Materials Science and Engineering, Chosun University, Gwangju 61452, South Korea \\ d Department of Chemistry, Kisan Veer Mahavidyalaya, Wai 412803, Maharashtra, India
}

\section{A R T I C L E I N F O}

\section{Keywords:}

Electrodeposition

Amorphous material

Electrocatalyst

HER

Selenide

\begin{abstract}
A B S T R A C T
The search for effective electrocatalysts for energy conversion is crucial for the advancement of green energy. Electrodeposition is a simple but effective method of preparing improved electrodes for use in the electrochemical sector. In the present work, we have synthesized amorphous NiMoSe nanospheres on Ni foam (NF) via a simple electrodeposition method. The synthesized NiMoSe demonstrates outstanding catalytic activity for the hydrogen evolution reaction (HER), with a small overpotential of $130 \mathrm{mV}$ at a current density of $10 \mathrm{~mA} \mathrm{~cm}-2$ in $1 \mathrm{M}$ $\mathrm{KOH}$ electrolyte and a small Tafel slope of $59 \mathrm{mV} \mathrm{dec}^{-1}$. Furthermore, NiMoSe also shows long-term durability over $45 \mathrm{~h}$.
\end{abstract}

\section{Introduction}

Hydrogen is recognized as the perfect energy carrier for the replacement of fossil fuels. Currently, methane reforming is used to produce hydrogen, which results in a huge emission of greenhouse gases and high energy consumption leading to global warming [1,2]. Electrolysis can be used to extract $\mathrm{H}_{2}$ from water, resulting in a cleaner manner of manufacturing. Water splitting is made up of two half-reactions: the HER and the oxygen evolution reaction (OER) $[3,4]$. While HER is a relatively easy process, OER has sluggish kinetics. In general, platinumgroup metals, are often used as electrocatalysts to improve the kinetics of the HER [5]. Nevertheless, their applications in large-scale commercialization suffer from supply shortages and expensive costs [6,7]. In this regard, it is extremely desirable to seek out low-cost non-noble metal-based catalysts with good electrocatalytic activity as viable HER electrocatalysts [8].

Transition metal selenides (TMSs) have demonstrated higher electron transport capabilities and improved electrochemical catalytic performances as a result of bigger atomic radii and regulated orbital occupation [9]. Moreover, TMSs show superior electrocatalytic activity than transition metal oxides due to the high conductivity of metallic Se compared to $\mathrm{O}[10,11]$. As a result, the electrocatalytic community has focused a lot of attention on TMSs [12].
Herein, we presented a facile strategy for the fabrication of NiMoSe nanospheres on conductive NF substrate via electrodeposition method. The obtained trimetallic NiMoSe nanosphere electrode exhibits excellent HER catalytic properties with an overpotential of $130 \mathrm{mV}$ to achieve current density of $10 \mathrm{~mA} \mathrm{~cm}{ }^{-2}$, a small Tafel slope of $59 \mathrm{mV}$ $\mathrm{dec}^{-1}$, and superior long-term stability for over $45 \mathrm{~h}$ in an alkaline medium. The high HER activity of NiMoSe electrode was attributed to a large number of electrochemically active surface areas and more active regions.

\section{Results and discussion}

The color of the NF changed from metallic to black during the electrodeposition procedure (detail process in supporting information). Fig. 1 (a) depicts the XRD pattern of NiMoSe electrode. The diffraction peaks at 44.5, 51.9, and 76.4 are attributed to the (1 111 ), ( $\left.\begin{array}{lll}2 & 0 & 0\end{array}\right)$, and (2 20 ) crystal planes of NF substrate, respectively [13]. There were no new diffraction peaks seen following NiMoSe electrodeposition, indicating that the NiMoSe deposited onto NF was amorphous. Fig. S1 shows the XPS survey spectra of the NiMoSe sample in which three elements, namely $\mathrm{Ni}, \mathrm{Mo}$, and Se are identified. Besides $\mathrm{O}$ was also observed in the XPS survey spectrum because of surface oxidation of selenium when exposed to air. The high-resolution Ni 2p XPS spectrum (Fig. 1(b)) shows

\footnotetext{
* Corresponding author.

E-mail address: jinhyeok@chonnam.ac.kr (J. Hyeok Kim).
} 

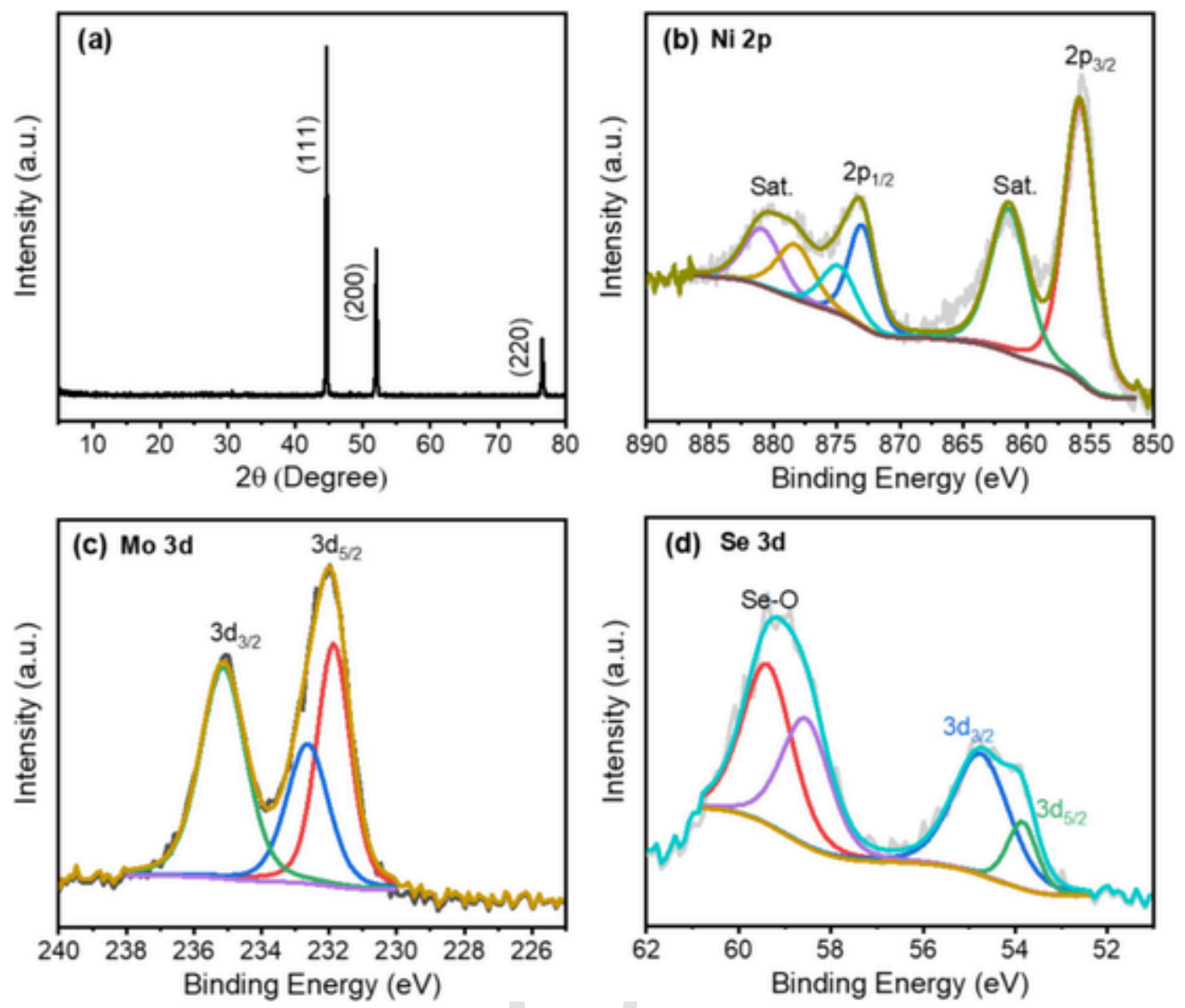

Fig. 1. (a) XRD pattern of NiMoSe, XPS spectra of (b) Ni 2p, (c) Mo 3d, (d) Se 3d of NiMoSe sample.

two main distinctive peaks at 856.1 and $874.1 \mathrm{eV}$, with matching satellite peaks ascribed to $\mathrm{Ni} 2 \mathrm{p}_{3 / 2}$ and $\mathrm{Ni} 2 \mathrm{p}_{1 / 2}$, respectively [14]. As shown in Fig. 1(c) Mo 3d high-resolution spectra show two distinct peaks at 231.9 and $235.1 \mathrm{eV}$ ascribed to $3 \mathrm{~d}_{5 / 2}$ and $3 \mathrm{~d}_{3 / 2}$, respectively, suggesting the presence of $\mathrm{Mo}^{6+}$ species. Simultaneously, the binding energy of Mo 3d changes to the left, slightly higher than the binding energy of pure Mo element (231.1 eV), showing that electron transfer occurs between Mo and Se [15]. The core level Se 3d XPS spectrum shows two peaks at 54.7 and $59.2 \mathrm{eV}$, corresponding to Se $3 \mathrm{~d}$ and Se-O, respectively. The Se $3 d$ could be deconvoluted into two peaks, $3 d_{3 / 2}$ and $3 d_{5 / 2}$ as seen in Fig. 1(d) [14].

The FE-SEM images of the NiMoSe sample at different magnifications are shown in Fig. 2(a, b). The as-deposited NiMoSe shows nanospheres decorated on NF substrate. The MoSe and NiSe samples also show same nanosphere morphology (Fig. S2(a, b)). Elemental mapping images of NiMoSe sample demonstrate a uniform distribution of $\mathrm{Ni}$, Mo, and Se throughout the nanosphere as shown in Fig. 2(c).

\subsection{Electrochemical performance for HER}

Fig. 3(a) shows the LSV curves for HER of all samples. The NiMoSe requires a low overpotential of $130 \mathrm{mV}$ to achieve a current density of $10 \mathrm{~mA} \mathrm{~cm}{ }^{-2}$, which is substantially lower than the MoSe (176 mV), NiSe $(211 \mathrm{mV})$, and the Bare NF $(229 \mathrm{mV})$. Tafel curves were plotted to reveal the reaction kinetics of the electrocatalysts, as shown in Fig. 3(b) [16]. The NiMoSe electrode possesses a much lower Tafel slope of $59 \mathrm{mV} \mathrm{dec}-1$, than that of MoSe $\left(71 \mathrm{mV} \mathrm{dec}^{-1}\right)$, NiSe $\left(97 \mathrm{mV} \mathrm{dec}{ }^{-1}\right.$ ), and Bare NF (116 mV dec $\left.{ }^{-1}\right)$ suggesting rapid HER kinetics of NiMoSe. Comparative plot of overpotential and Tafel values of all electrocata- lysts is plotted in Fig. 3(c). Fig. 3(d) displays stability test of NiMoSe electrode at constant current density of $10 \mathrm{~mA} \mathrm{~cm}^{-2}$ for $45 \mathrm{~h}$. The inset in Fig. 3(d) shows negligible change in overpotential after long-term stability. The FE-SEM and XPS were studied after stability. Fig. S3 shows the nanosphere morphology of NiMoSe remains same even after $45 \mathrm{~h}$ of continuous gas evolution. XPS analysis of NiMoSe after HER shows the presence of $\mathrm{Ni}$, Mo, and Se elements (Fig. S4).

To find out why NiMoSe has better electrocatalytic activity, the ECSA was calculated from the electrochemical double-layer capacitance $\left(\mathrm{C}_{\mathrm{dl}}\right)$ using typical $\mathrm{CV}$ data at different scan rates in the nonfaradaic zone (Fig. 4(a) and Fig. S5(a-b)) [17]. As shown in Fig. 4(b), the $\mathrm{C}_{\mathrm{dl}}$ of NiMoSe is $5.30 \mathrm{mF} \mathrm{cm}-2$, which is more than that of MoSe (3 $\mathrm{mF} \mathrm{cm}{ }^{-2}$ ) and NiSe $\left(1.98 \mathrm{mF} \mathrm{cm}^{-2}\right)$, indicating the presence of a greater number of electrochemically active sites in the NiMoSe. From the EIS spectra of all the electrodes as shown in Fig. 4(c) it is confirmed that NiMoSe electrode has smaller charge transfer resistance than the other two electrodes. Fig. S6 displays corresponding EIS fitting circuit of NiMoSe. Mass activity and specific activity of NiMoSe are $74.2 \mathrm{~mA} \mathrm{mg}^{-1}$ and $1.94 \mathrm{~mA} \mathrm{~cm}^{-2}$, respectively, whereas MoSe have $42.5 \mathrm{~mA} \mathrm{mg}^{-1}$ and $1.81 \mathrm{~mA} \mathrm{~cm}^{-2}$, and NiSe have $27.81 \mathrm{~mA} \mathrm{mg}^{-1}$ and $1.34 \mathrm{~mA} \mathrm{~cm}{ }^{-2}$.

\section{Conclusion}

In summary, NiMoSe nanospheres were successfully synthesized via a simple electrodeposition technique on NF in a short time for the application of HER. Benefitting from the large electrochemically active surface area, small charge-transfer resistance the NiMoSe electrode show excellent catalytic activity for HER with a low overpotential of $130 \mathrm{mV}$ to achieve a current density of $10 \mathrm{~mA} \mathrm{~cm}^{-2}$ as well as small Tafel slope 


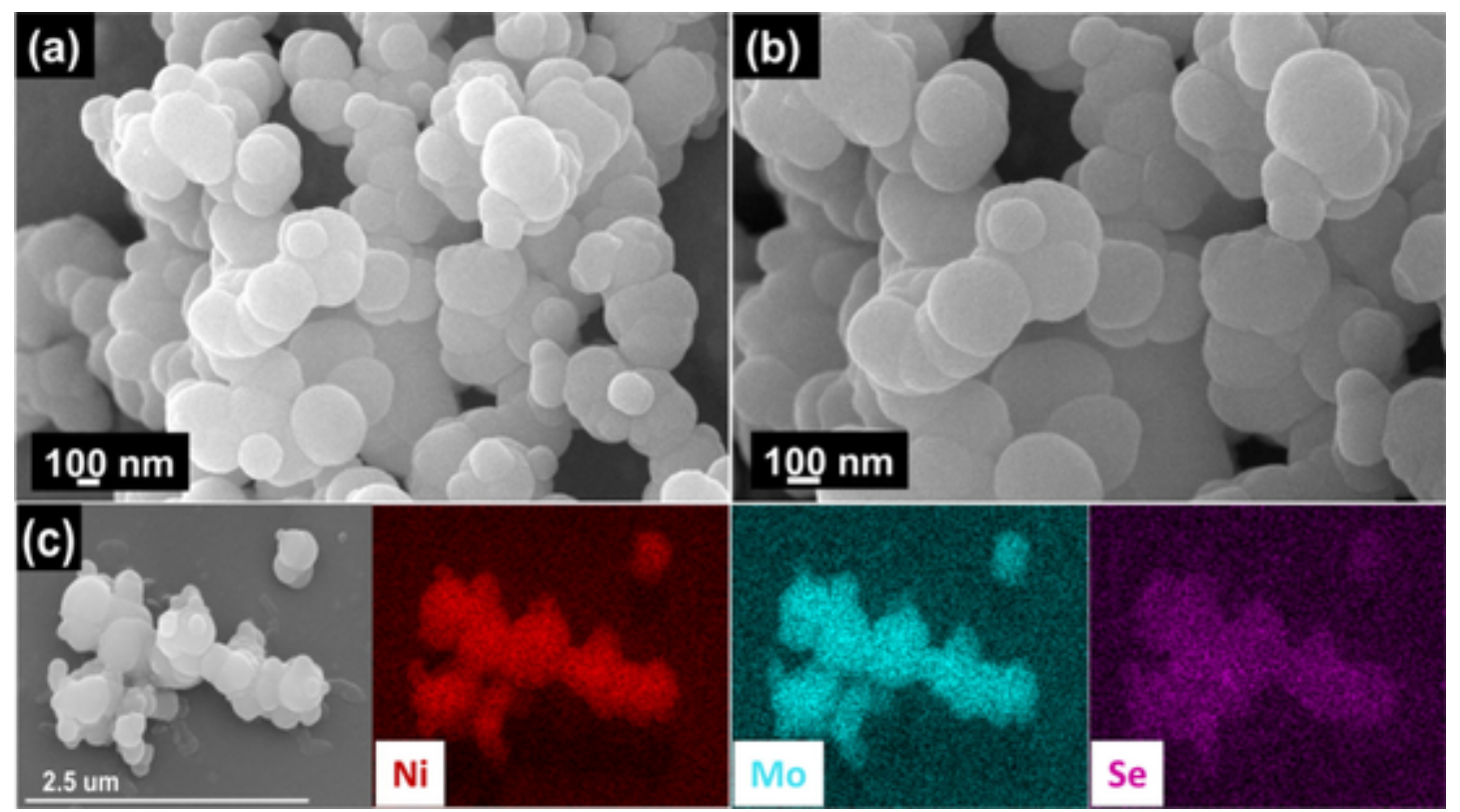

Fig. 2. (a and b) FE-SEM images of NiMoSe, (c) Corresponding elemental mappings of Ni, Mo, Se elements.
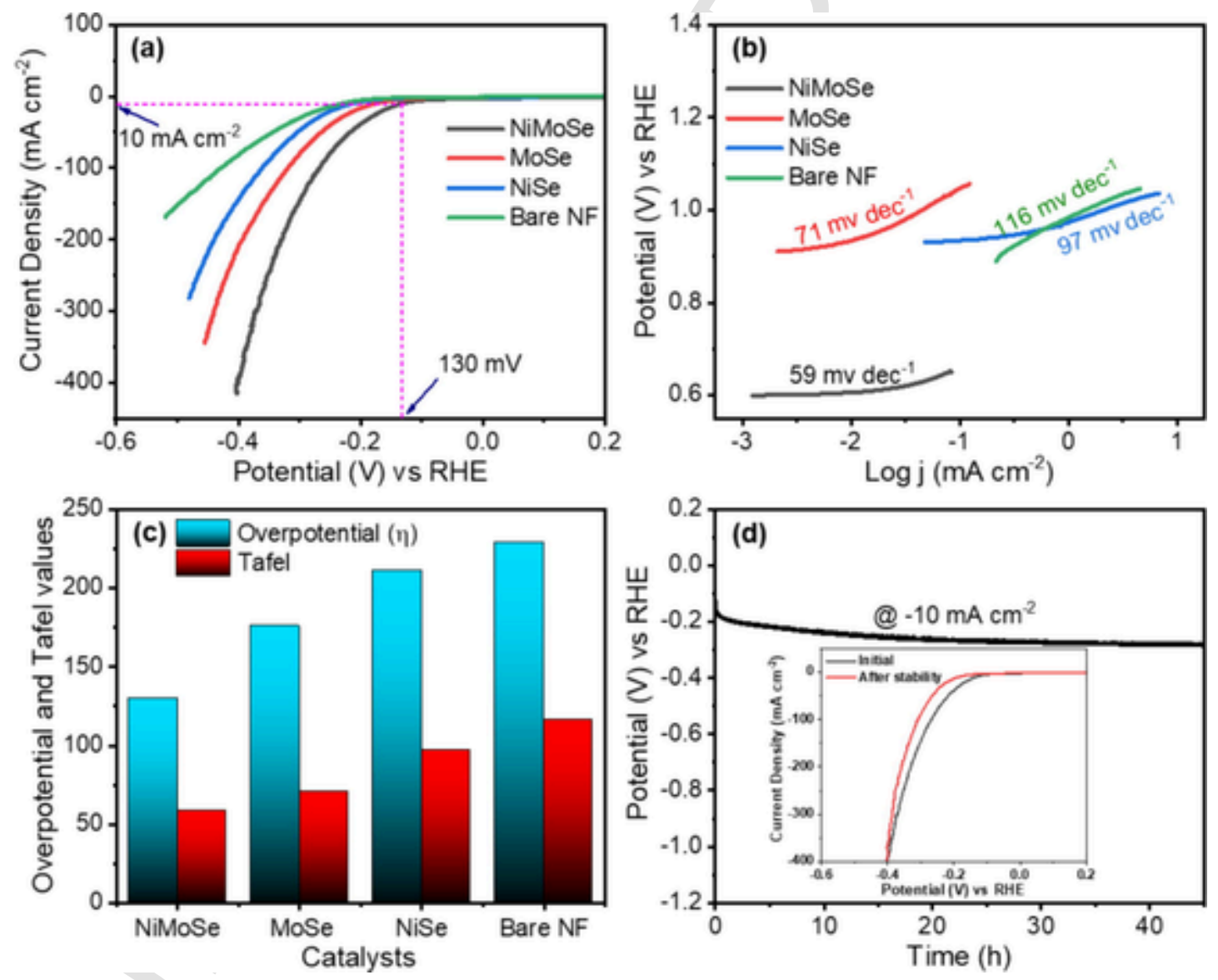

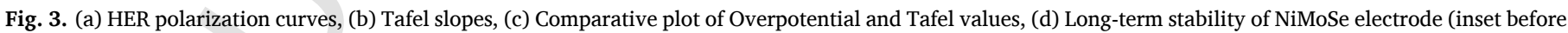
and after stability LSV curve).

of $59 \mathrm{mV} \mathrm{dec}{ }^{-1}$. More importantly, the NiMoSe exhibits excellent stability for $45 \mathrm{~h}$ in $1 \mathrm{M} \mathrm{KOH}$. As a result, electrodeposited NiMoSe may be an excellent choice for use as a platinum-free electrocatalyst in the water-splitting process.

\section{CRediT authorship contribution statement}

Komal Patil: Formal analysis, Data curation, Writing - original draft. Pravin Babar: Writing - original draft, Writing - review \& editing, Resources. Xue Li: Methodology, Software. Junsung Jang: Software, Data curation. Dongmyeong Kim: Data curation, Formal 

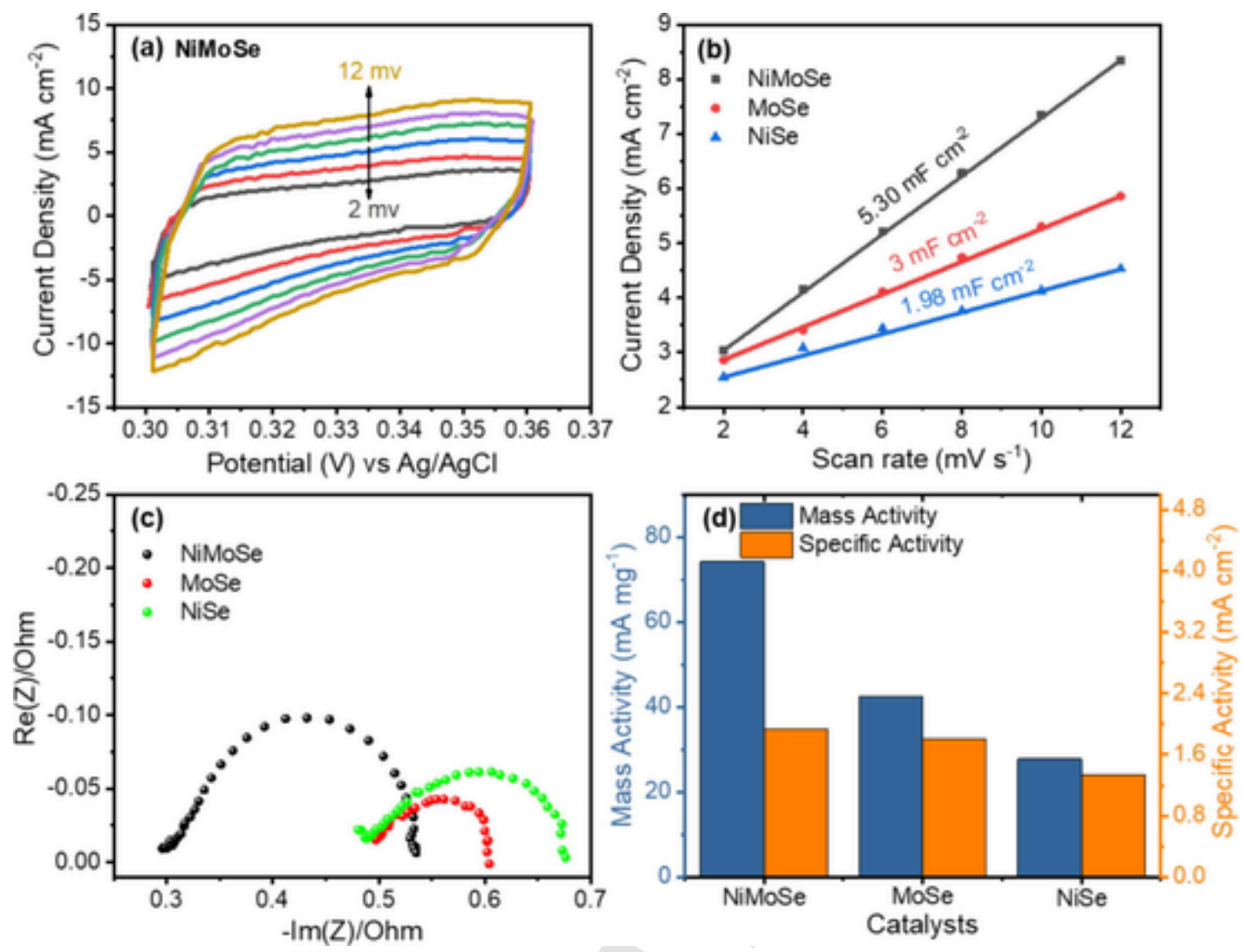

Fig. 4. (a) CV curves of NiMoSe at different scan rates, (b) Current density as a function of scan rate, (c) EIS spectra, (d) Mass and specific activities of catalysts at $350 \mathrm{mV}$ overpotential.

analysis. Myeong Cheol Baek: Formal analysis, Software. Pravin Bhoite: Supervision. Jin Hyeok Kim: Funding acquisition, Project administration, Supervision.

\section{Declaration of Competing Interest}

The authors declare that they have no known competing financial interests or personal relationships that could have appeared to influence the work reported in this paper.

\section{Acknowledgments}

This work was supported by the Human Resources Development Program (No. 20194030202470) of the Korea Institute of Energy Technology Evaluation and Planning (KETEP) Grant funded by the Korean Government Ministry of Trade, Industry and Energy. This work was supported by the Technology Development Program to Solve Climate Changes of the National Research Foundation (grant no. 2016M1A2A2936784) funded by the Ministry of Science and ICT.

\section{Appendix A. Supplementary data}

Supplementary data to this article can be found online at https:// doi.org/10.1016/j.matlet.2021.131409.

\section{References}

[1] B. You, M.T. Tang, C. Tsai, F. Abild-Pedersen, X. Zheng, H. Li, Adv. Mater. 31 (2019) 1807001

[2] P. Babar, K. Patil, V. Karade, K. Gour, A. Lokhande, S. Pawar, J.H. Kim, ACS Appl. Mater. Interfaces. 13 (2021) 52620-52628.

[3] P. Babar, K. Patil, D.M. Lee, V. Karade, K. Gour, S. Pawar, J.H. Kim, J. Colloid Interface Sci. 584 (2021) 760-769.

[4] K. Patil, P. Babar, D.M. Lee, V. Karade, E. Jo, S. Korade, J.H. Kim, Sustain. Energy Fuels 4 (2020) 5254-5263.

[5] M. Cao, Z. Xue, J. Niu, J. Qin, M. Sawangphruk, X. Zhang, R. Liu, ACS Appl. Mater. Interfaces 10 (2018) 35224-35233.

[6] J. Niu, Y. Yue, C. Yang, Y. Wang, J. Qin, X. Zhang, Z.S. Wu, Appl. Surf. Sci. 561 (2021) 150030.

[7] M. Cao, X. Zhang, J. Qin, R. Liu, ACS Sustain. Chem. Eng. 6 (2018) 16198-16204.

[8] P. Babar, A. Lokhande, V. Karade, I.J. Lee, D. Lee, S. Pawar, J.H. Kim, J. Colloid Interface Sci. 557 (2019) 10-17.

[9] W.-F. Chen, K. Sasaki, C. Ma, A.I. Frenkel, N. Marinkovic, J.T. Muckerman, Y. Zhu, R.R. Adzic, Angew. Chem. Int. Ed. 51 (2012) 6131-6135.

[10] J. Tian, N. Cheng, Q. Liu, X. Sun, Y. He, A.M. Asiri, J. Mater. Chem. A. 3 (2015) 20056-20059.

[11] Y. Liu, H. Cheng, M. Lyu, S. Fan, Q. Liu, W. Zhang, Y. Zhi, C. Wang, C. Xiao, S. Wei, B. Ye, Y. Xie, J. Am. Chem. Soc. 136 (2014) 15670-15675.

[12] A. Sivanantham, S. Shanmugam, Appl. Catal. B Environ. 203 (2017) 485-493.

[13] P. Babar, A. Lokhande, V. Karade, B. Pawar, M.G. Gang, S. Pawar, J.H. Kim, ACS Sustain. Chem. Eng. 7 (2019) 10035-10043.

[14] H. Wang, X. Jiao, W. Zeng, Y. Zhang, Y. Jiao, Int. J. Hydrogen Energy (2021).

[15] Y. Lu, S. Geng, S. Wang, S. Rao, Y. Huang, X. Zou, Y. Zhang, Q. Xu, X. Lu, Int. J. Hydrogen Energy 44 (2019) 5704-5716.

[16] S.M. Pawar, B.S. Pawar, P.T. Babar, A.T. Aqueel Ahmed, H.S. Chavan, Y. Jo, S. Cho, J. Kim, A.I. Inamdar, J.H. Kim, H. Kim, H. Im, Mater. Lett. 241 (2019) 243-247.

[17] K. Patil, J.H. Kim, Korean J. Mater. Res. 30 (2020). 